\title{
Características Del Buen Profesor De Modalidad Presencial Y Virtual Desde La Perspectiva De Los Estudiantes
}

\author{
Mirsha Alicia Sotelo Castillo \\ Javier José Vales García \\ Ramona Imelda García López \\ Laura Fernanda Barrera Hernández \\ Instituto Tecnológico de Sonora, México
}

doi: 10.19044/esj.2017.v13n13p78 URL:http://dx.doi.org/10.19044/esj.2017.v13n13p78

\begin{abstract}
The purpose of the study was to identify the characteristics associated to the good teacher who teaches presential and virtual mode. A total of 713 students participated, distributed in two groups (493 and 220), from different academic programs of a Mexican institution of higher education participated. For data collection, the technique of semantic networks was used, they were presented as stimulus phrase "good presential teacher" and "good virtual teacher". Two semantic networks were obtained, one for presential teacher, generating 269 definers and a network core of 39. The other semantic network was for the virtual teacher, where 118 words and a network nucleus of 37 were generated. The three words that resulted in greater semantic weight were: responsible, respectful and comprehensive for the presential, accessible and available for the virtual. This study concludes that the definition the students do of the good teacher, regardless of the modality, is determined by personal values.
\end{abstract}

Keywords: Professors, students, presential mode, virtual mode, semantic networks

\section{Resumen}

El propósito del estudio fue identificar las características asociadas al buen profesor que imparte asignaturas en modalidad presencial y virtual. Participaron en total 713 estudiantes, distribuidos en dos grupos (493 y 220), de diferentes programas académicos de una institución mexicana de educación superior. Para la recolección de los datos se utilizó la técnica de redes semánticas en donde se les presentó como frase estímulo "buen 
profesor presencial" y "buen profesor virtual". Se obtuvieron dos redes semánticas, una para el profesor presencial, generándose 269 palabras definidoras y un núcleo de red de 39; la otra, fue para el profesor virtual, en donde se generaron 118 palabras y un núcleo de red de 37. Las tres palabras que resultaron con un mayor peso semántico fueron responsable, respetuoso y comprensivo para el profesor presencial y responsable, accesible y disponible para el virtual. Con este estudio se concluye que la definición del buen profesor que hacen los estudiantes, independientemente de la modalidad, está determinada por valores personales.

Palabras clave: Profesores, estudiantes, modalidad presencial, modalidad virtual, redes semánticas

\section{Introducción}

El estudio de las características de un buen profesor universitario ha sido investigado por diferentes autores. Fernández y González (2012) mencionan que para lograr un buen aprendizaje en los estudiantes es importante que el docente promueva el desarrollo de competencias, de manera que se garantice el aprendizaje continuo y autónomo; bajo este supuesto, la figura o el papel que desempeña el docente frente a los estudiantes es de suma importancia. Ayala y Cabrera (2010) consideran como modelos básicos de formación docente: la adquisición de conocimientos innovadores, el desarrollo de habilidades del rendimiento didáctico, su autoconcepto y la investigación en el aula, buscando soluciones a los problemas presentes en el contexto educativo; y como competencias necesarias para la docencia, el dominio de la asignatura impartida y de la cultura, competencias pedagógicas (habilidades didácticas, tutoría, conocimientos psicológicos y sociales), habilidades instrumentales y nuevos conocimientos (tecnología, lenguajes, etcétera) y características personales (madurez, autoestima, empatía).

Los resultados del informe de investigación de Moreno (2011) sobre las creencias de lo que es un buen profesor indican que los docentes contemplan entre sus competencias o habilidades: ser consciente de su profesión, autonomía, capacidad de transmitir, motivar y ponerse en el lugar del alumno, saber guiar, orientar y facilitar procesos de aprendizaje; por otra parte, los alumnos reportaron una alta importancia a las características personales.

Fernández y González (2012), con el propósito de describir el rol que debe desempeñar el profesor en el ámbito educativo de nivel universitario, desde la perspectiva del alumnado, mencionan que para los estudiantes la característica principal, de acuerdo a los resultados asociada a un buen profesor, con una media mayor fue el explicar con claridad, seguida de ser 
especialista en la materia que imparte, destacar las ideas principales y motivar al alumnado y preocuparse por el aprendizaje de los alumnos.

Asimismo, López, González y De León (2014), a partir del protocolo aplicado a estudiantes para validar un sistema de indicadores de evaluación de la calidad de la actividad docente, concluyen que el perfil docente debe responder a un profesional que desarrolla su actividad de manera planificada y sistemática, donde la información a transmitir está ligada con las competencias profesionales del trabajo, emplea recursos, metodologías y estrategias didácticas en un contexto especifico de enseñanza-aprendizaje donde la planificación docente, la gestión del tiempo y del espacio, los sistemas de evaluación y la interacción con el alumnado juegan un papel importante.

Gargallo, Sánchez, Ros y Ferreras (2010) en su estudio con 50 estudiantes, encontraron que las habilidades o cualidades de un buen profesor, las cuales fueron agrupadas en las siguientes dimensiones:

- $\quad$ Personales: que incluyen el respeto a los alumnos, ser abierto, con capacidad de escucha, comprensivo y con carácter agradable.

- Profesionales: competencia, buena comunicación, preparación de clases y responsabilidad.

- $\quad$ Metodología empleada por los docentes: que enseñe estrategias para trabajar la asignatura y aprender (técnicas de estudio, preparación de exámenes, hablar en público), establezca relaciones entre los conceptos y temas de la asignatura, fomente la participación e implicación de los alumnos y utilice los recursos de apoyo necesarios (power point, transparencias clásicas, vídeos, etc).

- Características de las explicaciones: motivadoras, haciendo uso de ejemplos prácticos y reales, relacione teoría y práctica y sean claras; métodos: debe generar aprendizaje significativo (no memorístico, estableciendo relaciones entre lo nuevo y lo viejo), método socráticomayéutico (explicación, preguntas, resolución de problemas, construyendo críticamente la materia con la participación de los alumnos) y explicación de un contenido de diversas maneras (uso de metodologías variadas de enseñanza).

- $\quad$ Materiales o recursos: claros y sencillos, apuntes de calidad ajustados a lo que hay que aprender, modelos de examen, además del uso de material complementario para el aprendizaje (esquemas, resúmenes, fotocopias).

- $\quad$ Métodos de evaluación: realizar una evaluación formativa donde el alumno conozca sus fallos y limitaciones y pueda corregirlos, valorando no sólo la memorización, sino el razonamiento y el aprendizaje significativo y continua; es decir, de forma periódica y constante.

Algunos estudios han utilizado la técnica de redes semánticas para definir a un buen profesor. Cabalín y Navarro (2008) obtuvieron como 
resultados de su estudio, a las palabras "respetuoso" y "responsable", seguidas por "comprensivo", "empático", "puntual”, "inteligente", "amable", "claro", "organizado" y "motivador" como descriptores del buen profesor; además realizaron comparaciones respecto a las carreras estudiadas, encontrándose como palabra descriptora "responsable", en primer orden en 4 de las 7 carreras analizadas, y en todas se mostraba dicha palabra y "respetuoso", en alguno de los 3 primeros descriptores. Finalmente, estos autores concluyeron que las principales palabras descriptoras del buen profesor universitario indicadas por los alumnos se refieren a los aspectos personales del docente y no a situaciones referentes a características relacionadas al conocimiento disciplinar, ni relativas al manejo pedagógico. Posteriormente Cabalín, Navarro, Zamora y Martín (2010) confirmaron nuevamente los hallazgos de Cabalín y Navarro (2008); sin embargo, además sumaron resultados provenientes de profesores, quienes indicaron que un buen profesor es responsable, empático, con conocimiento, comprometido, actualizado y estudioso.

\section{Perfil del profesor en modalidad virtual}

La educación ha evolucionado, debido, entre otras razones, a los avances tecnológicos lo que ha generado muchos desafíos y cambios en el sistema educativo. Al respecto, González, Padilla y Rincón (2011) mencionan que el reto más fuerte lo afrontan los maestros, ya que su función docente se ve transformada; los métodos tradicionales de enseñanza han sido modificados y por tanto, el perfil docente actual contempla características, habilidades y competencias nuevas, donde generalmente se incluye el manejo adecuado y provechoso de herramientas tecnológicas y la producción de materiales (Suárez \& Fernández, 2013). Ardila (2010) atribuye a los profesores responsabilidades a nivel técnico y educacional; mientras que Cabero (2004) afirma que enseñar no es sólo proporcionar información, sino que el docente debe ayudar a aprender; para tal logro, debe tener un buen conocimiento de sus alumnos, cuáles son sus ideas previas, capacidad de aprendizaje, estilos de aprendizaje, motivaciones, actitudes, valores, entre otros.

Al respecto, se han realizado estudios desde las perspectiva de los estudiantes para conocer las habilidades, conocimientos y actitudes del profesor en modalidad virtual. Del Hierro, García y Mortis (2014) encontraron que los alumnos señalan como importante el dominio de habilidades tecnológicas, predominando la utilización de la plataforma tecnológica y la publicación de materiales de utilidad para la asignatura en la misma plataforma; asimismo, señalan como necesario que el docente promueva la comunicación en el curso, y sea un agente motivador para promover actividades que apoyan la autorregulación del aprendizaje por el 
propio alumno. Lo anterior concuerda con lo encontrado por Imbernón, Silva y Guzmán (2011), quienes mencionan que las competencias de los profesores que imparten cursos en modalidad virtual o mixta son: conocimiento y utilización de las herramientas informáticas y telemáticas; conocimiento y utilización de técnicas de planificación y diseño en el ámbito virtual; conocimiento y utilización de metodologías didácticas para el e-learning y el b-learning. Además, deben incluir en las competencias docentes el diseño de materiales, el dominio de contenidos y la planificación del proceso de aprendizaje (Blázquez \& Alonso, 2009).

Dado lo anterior y considerando los diferentes estudios, se esperaría que en el perfil del docente en modalidad virtual, estuviera presente el dominio pedagógico y tecnológico; por ello se considera necesario desarrollar este trabajo con el objetivo de identificar, desde la perspectiva de los estudiantes, cuáles son las características asociadas al buen profesor en modalidad presencial y virtual y analizar sus diferencias.

\section{Método}

\section{Participantes}

En total participó un total de 713 estudiantes universitarios distribuidos en dos grupos, a continuación se describen las características de cada grupo de participantes.

El primer grupo se formó por 493 estudiantes, fueron seleccionados de manera intencional y pertenecían a diferentes programas académicos de una institución pública mexicana de nivel superior (279 hombres y 214 mujeres). Éstos tuvieron una media de edad de 21 años. A este grupo se le preguntó sobre las características del profesor universitario en modalidad presencial.

Para la recolección de datos sobre las características del profesor virtual participaron 220 estudiantes (79 hombres y 141 mujeres). La media de edad para los participantes fue de 20 años. La muestra se seleccionó de manera intencional, el único criterio de inclusión fue que los alumnos hubieran tomado, por lo menos un curso en modalidad virtual o mixta.

\section{Instrumento}

Para la recolección de datos se aplicó la técnica de redes semántica (Vera-Noriega, Pimental \& Batista, 2005) utilizando como palabra estímulo "buen profesor presencial", en caso del docente en modalidad presencial y "buen profesor virtual", para el caso de la modalidad virtual, esto con la finalidad de conocer la percepción de los estudiantes sobre dicho concepto, así como el vocabulario utilizado para referirse a éste. Para ello, se solicitó que en un formato escribieran 5 palabras que asociaran con la frase estímulo; posteriormente se les solicitó jerarquizar según el orden de importancia del 1 
al 5, tomando en cuenta que el 1 es el más importante y el 5, el menos importante.

\section{Procedimiento y análisis de datos}

Primeramente se eligió la muestra de manera intencional, la cual quedó conformada por estudiantes de distintas áreas de conocimiento. La aplicación se llevó a cabo en las aulas de la institución, previa autorización de los maestros y una vez obtenida se procedió a la aplicación, iniciando con el objetivo e instrucciones de los instrumentos. El análisis de la red semántica se realizó en el programa Microsoft Excel 2010, donde se calculó el tamaño de red (TR), el peso semántico (PS), el núcleo de red (NR) y la distancia semántica cuantitativa (DSC).

\section{Resultados}

Referente al perfil del buen profesor universitario (en modalidad presencial) el grupo de estudiantes universitarios produjeron un tamaño de red (TR) correspondiente a 269 palabras definidoras. Al realizar la suma de la ponderación de la frecuencia por la jerarquización asignada por el grupo para obtener el peso semántico, se incluyeron en el núcleo de red 39 definidoras sin perder su representatividad de significancia, correspondiendo el último lugar a la palabra de disponibilidad; la definidora 40 ya no se incluyó puesto que fue el punto de quiebre donde el peso semántico se vuelve asintótico (ver figura 1).

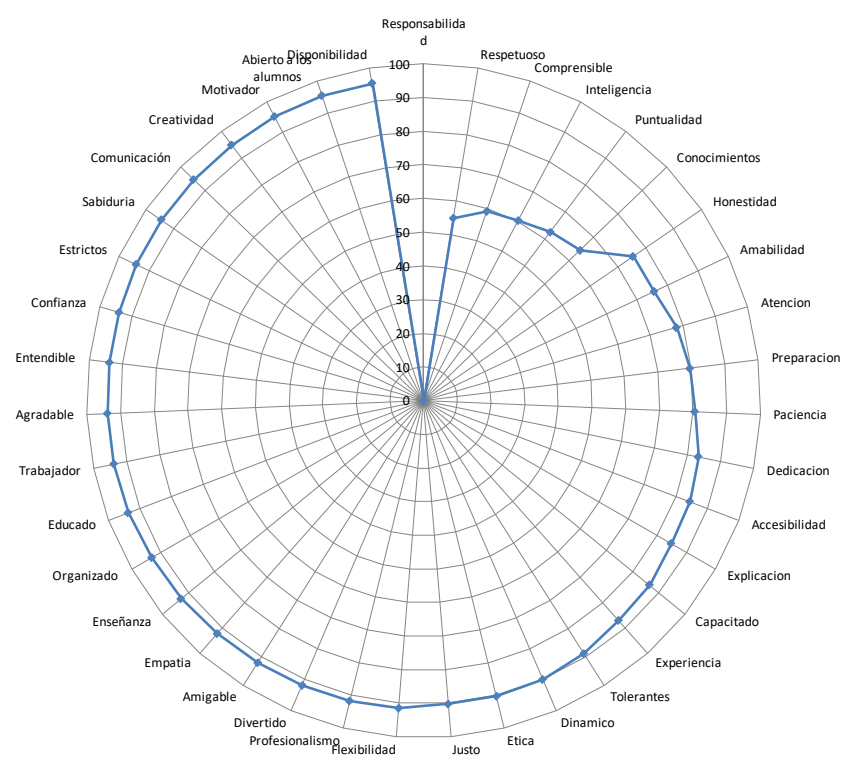

Figura 1. Red obtenida de las definidoras de "buen profesor presencial" 
Más del $50 \%$ de los estudiantes coinciden en la palabra responsabilidad como la principal definidora de un buen profesor, seguida por respetuoso (45\%), comprensivo (41\%), inteligencia (40\%), puntualidad (37\%) y conocimientos (36\%). En la tabla 1 se puede observar el total de las palabras que fueron incluidas en el conjunto de definidoras.

Tabla 1. Peso y distancia semántica del conjunto de definidoras del buen profesor presencial

\begin{tabular}{cccccc}
\hline Definidora & PS & \% DS & Definidora & PS & \% DS \\
\hline Responsabilidad & 2289 & 100 & Flexibilidad & 194 & 8 \\
Respetuoso & 1033 & 45 & Profesionalismo & 189 & 8 \\
Comprensivo & 934 & 41 & Divertido & 187 & 8 \\
Inteligencia & 908 & 40 & Amigable & 183 & 8 \\
Puntualidad & 858 & 37 & Empatía & 176 & 8 \\
Conocimientos & 816 & 36 & Enseñanza & 162 & 7 \\
Honestidad & 565 & 25 & Organizado & 158 & 7 \\
Amabilidad & 559 & 24 & Educado & 143 & 6 \\
Atención & 505 & 22 & Trabajador & 143 & 6 \\
Preparación & 470 & 21 & Agradable & 141 & 6 \\
Paciencia & 449 & 20 & Entendible & 141 & 6 \\
Dedicación & 388 & 17 & Confianza & 136 & 6 \\
Accesibilidad & 358 & 16 & Estrictos & 129 & 6 \\
Explicación & 351 & 15 & Sabiduría & 125 & 5 \\
Capacitado & 312 & 14 & Comunicación & 122 & 5 \\
Experiencia & 296 & 13 & Creatividad & 117 & 5 \\
Tolerantes & 257 & 11 & Motivador & 110 & 5 \\
Dinámico & 231 & 10 & Abierto a los alumnos & 105 & 5 \\
Ética & 222 & 10 & Disponibilidad & 105 & 5 \\
Justo & 222 & 10 & & & \\
\hline
\end{tabular}

Con respecto al perfil del buen profesor universitario en modalidad virtual, se produjo un TR correspondiente a 118 palabras definidoras. Al realizar la suma de la ponderación de la frecuencia por la jerarquización asignada por el grupo para obtener el PS, se incluyeron en el NR 37 definidoras sin perder su representatividad de significancia, correspondiendo el último lugar a la palabra de disponibilidad (ver figura 2); la definidora 38 ya no se incluyó puesto que fue el punto de quiebre donde el PS se vuelve asintótico. 


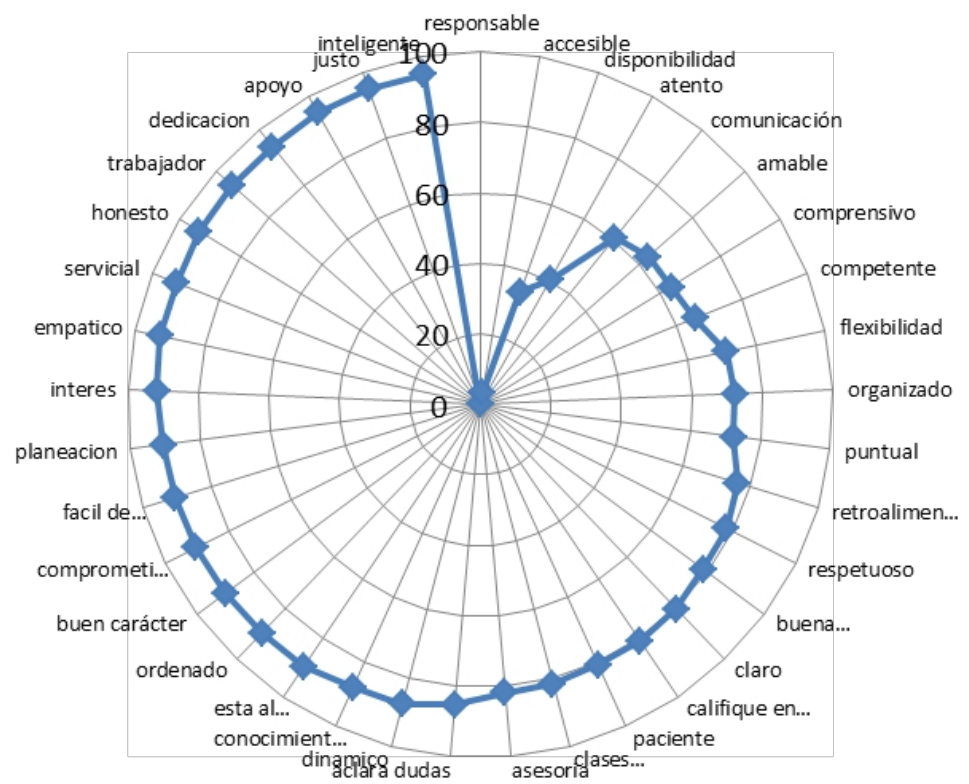

Figura 2. Red obtenida de las definidoras de "buen profesor virtual"

El 100\% de los participantes coincidieron en la palabra responsable como la principal definidora de un buen profesor en ambientes virtuales, seguida por accesible (96\%), disponibilidad (66\%), atento (59\%), comunicación (39\%) y amable (36\%). En la tabla 2 se puede observar el total de las palabras que fueron incluidas en el conjunto de definidoras así como el peso semántico y la distancia semántica en porcentaje.

Tabla 2. Peso y distancia semántica del buen profesor virtual

\begin{tabular}{cccccc}
\hline Definidora & PS & \% DS & Definidora & PS & \% DS \\
\hline Responsable & 813 & 100 & Aclara dudas & 119 & 14.64 \\
Accesible & 785 & 96.56 & Dinámico & 99 & 12.18 \\
Disponibilidad & 537 & 66.05 & Habilidades tecnológicas & 98 & 12.05 \\
Atento & 484 & 59.53 & Esté al pendiente & 84 & 10.33 \\
Comunicación & 320 & 39.36 & Ordenado & 84 & 10.33 \\
Amable & 297 & 36.53 & Buen carácter & 82 & 10.09 \\
Comprensivo & 296 & 36.41 & Comprometido & 78 & 9.59 \\
Competente & 280 & 34.44 & Fácil de localizar & 76 & 9.35 \\
Flexibilidad & 233 & 28.66 & Planeación & 75 & 9.23 \\
Organizado & 225 & 27.68 & Interés & 66 & 8.12 \\
Puntual & 225 & 27.68 & Empático & 58 & 7.13 \\
Retroalimentación & 194 & 23.86 & Servicial & 58 & 7.13 \\
Respetuoso & 178 & 21.89 & Honesto & 52 & 6.40 \\
Buena explicación & 173 & 21.28 & Trabajador & 50 & 6.15 \\
Claro & 161 & 19.80 & Dedicación & 49 & 6.03 \\
Califique en tiempo y forma & 156 & 19.19 & Apoyo & 42 & 5.17 \\
Paciente & 155 & 19.07 & Justo & 42 & 5.17 \\
Clases presenciales & 148 & 18.20 & Inteligente & 41 & 5.04 \\
Asesoría & 147 & 18.08 & & &
\end{tabular}




\section{Discusión}

Las primeras tres palabras definidoras sobre las características que debe poseer el buen profesor en modalidad presencial están relacionadas con valores personales del profesor; al respecto Cabalín y Navarro (2008) encontraron resultados similares, en donde las palabras respetuoso y responsable están relacionadas directamente con el buen profesor. Además también se mencionan los conceptos de puntualidad, honestidad, amabilidad, atención, paciencia, lo que hace alusión al trato que desean recibir por parte de los docentes; esto mismo coincide con lo presentado por Cataldi y Lage en el 2004.

Es importante señalar, ya que era una característica esperada, que los aspectos relacionados con el desempeño docente (competencia) no destacaron en las definidoras de los estudiantes, como sucede en el estudio de Corona (2008), en donde se encontraron aspectos del área académica, conocimientos y preparación de la clase, además las expectativas también están centradas en las estrategias didácticas más que en los valores y actitudes.

En lo que respecta a las características de los profesores en modalidad virtual, hay una coincidencia en lo mencionado con los profesores presenciales, ya que los estudiantes consideran más importante que el docente debe ser responsable, accesible, que tenga disponibilidad, que sea atento, amable, comprensivo entre otras; estableciendo estas características en las categorías señaladas por Cabero (2004b), UNESCO (2008), Ardilla (2009), Padilla, Leal, Hernández y Cabero (2014) y Del Hierro, García y Mortis (2014), quienes coinciden en que existen dos competencias: formación intelectual operativa (incluye funciones académicas, pedagógicas, orientadoras y tecnológicas); y formación personal docente (valores y actitudes); la mayoría de las palabras se ubican en el aspecto actitudinal. Las habilidades tecnológicas las colocan hasta el lugar 23 y sólo hacen referencia a que tenga conocimiento de la plataforma.

Respecto a los conocimientos o dominio científico y pedagógico, indicaron que sean competentes, flexibles, que sean "buenos" para explicar, dinámicos e inteligentes. Cabe mencionar que la mayoría de las clasificaciones estudiadas sobre competencias de los profesores en ambientes virtuales se enfocan en el componente pedagógico y didáctico, en virtud de que las principales funciones que tiene el profesorado en dichos entornos son el diseño y el desarrollo de ambientes de aprendizaje que ayuden al estudiante en su proceso de formación. Al respecto García, Amaro y Brioli (2012) asumen que los docentes universitarios que desarrollan actividades formativas virtuales, tienen competencias básicas en las tecnologías de la información y la comunicación (TIC). Sin embargo, el dominio, la buena voluntad e iniciativa de aplicar las herramientas tecnológicas, no garantiza su 
uso adecuado desde el punto de vista didáctico en una modalidad mixta o virtual.

En los resultados de este estudio, llama la atención que los aspectos relacionados con el desempeño no destacan en las definidoras de los estudiantes, en ninguna de las modalidades (presencial y virtual), además en la modalidad virtual no es tan importante un dominio tecnológico, ya que para ellos un buen profesor está determinado por atributos valóricos.

El conocer las características que hace a un buen profesor, tanto en ambientes presenciales como virtuales, desde la opinión de los estudiantes, permite reflexionar sobre la efectividad del proceso enseñanza-aprendizaje, además de orientar sobre el nivel de la calidad de la enseñanza en la institución, ya que permiten identificar indicadores que permitan establecer mejores prácticas docentes, así como desarrollar competencias y estrategias que se orienten a la consecución de una mejora del proceso de todas las partes implicadas.

\section{References:}

1. Ardila, M. (2009). Docencia en ambientes virtuales: nuevos roles y funciones. Revista Virtual Universidad Católica del Norte, 28, 1-15. Recuperado de http://www.redalyc.org/ articulo.oa?id=194214468004

2. Ayala, E. y Cabrera, R. (2010). ¿Qué hace a un docente, un buen docente? Trabajo presentado en el Cuarto Foro Nacional de Ciencias Básicas. México. Recuperado de http://dcb.fic.unam.mx/Eventos/Foro4/Memorias/Ponencia_12.pdf

3. Blázquez, F., y Alonso, L. (2009). Funciones del profesor de elearning. Revista de Medios y Educación, (34), 205-215. Recuperado de https://idus.us.es/xmlui/bitstream/ handle/11441/22586/file_1.pdf?sequence=1\&isAllowed=y

4. Cabalín, D., Navarro, N., Zamora, J., y Martín, S. (2010). Concepción de estudiantes y docentes del buen profesor universitario. Int. J. Morpho., 28(1), 283-290,

5. Cabalín, D., y Navarro, N. (2008). Conceptualización de los estudiantes sobre el buen profesor universitario en las carreras de la Salud de la Universidad de La Frontera. Chile. Int. J. Morphol, 26(4), 887-892. Recuperado de http://www.scielo.cl/pdf/ ijmorphol/v26n4/ art17.pdf

6. Cabero, J. (2004a). Las Web para la formación. En J. Salinas, J. Cabero-Almenara, y J. Aguaded. (Eds.), Tecnologías para la educación. Diseño, producción y evaluación de medios para la formación docente. Madrid: Alianza Editorial. 
7. Cabero, J. (2004b). La función tutorial en la teleformación. En F. Martínez y M. Prendes (Eds.), Nuevas Tecnologías y Educación. Madrid: Pearson Educación.

8. Cabero, J. (2014). La formación del profesorado en TIC: unas referencias conceptuales. En J. Silva y J. Salinas (Eds.), Innovando con TIC en la formación inicial docente: aspectos teóricos y casos concretos (pp. 173-192). Chile. Recuperado de: http://pfc.upnfm.edu.hn/

multiteca/documentos/libros/TIC_Tecnologias_Informacion_Comuni cacion/Innovando_con_TIC_en_la_formacion_inical_docente.pdf

9. Cataldi, Z. y Lage, F. (2004). Un nuevo perfil del profesor universitario. Revista De Informática Educativa y Medios Audiovisuales. 1(3):28-33, 2004.

10. Corona, C. (2008). Que hace el buen maestro?. La visión del estudiante de ciencias físico matemáticas. Lat. Am. J. Phys. Educ., 2(2):147-15.

11. Del Hierro, E., García, R., y Mortis, S. (2014). Percepción de estudiantes universitarios sobre el perfil del profesor en la modalidad virtual-presencial. EDUTEC. Revista Electrónica de Tecnología Educativa, (48). Recuperado de http://edutec.rediris.es/ Revelec2/ Revelec48/pdf/Edutec-e_n48_Del_Hierro.pdf

12. Fernández, M., y González, S. (2012). El perfil del buen docente universitario. Una aproximación en función del sexo del alumnado. Revista de Docencia Universitaria, 10(2), 237-249. Recuperado de red-u.net/redu/index.php/REDU/article/view/ 325/pdf

13. García, I.F., Amaro, R., y Brioli, C. (2012). La valoración del docente universitario en entornos virtuales: algunos Descriptores claves. Revista Historia de la Educación Latinoamericana, 14(19), 205-226. Recuperado de http://www.redalyc.org/ articulo.oa?id=86926976010

14. Gargallo, B., Sanchez, F., Ros, C., y Ferreras, A. (2010). Estilos docentes de los profesores universitarios. La percepción de los alumnos de los buenos profesores. Revista Iberoamericana de Educación, 51(4). Recuperado de http://rieoei.org/deloslectores/ 3236Lopez.pdf

15. González, G., Padilla, B. y Rincón, C. (2011). Fundamentos conceptuales para a evaluación del docdene en contextos b-learning. Revista Virtual Universidad Católica del Norte, 34, 220-243. Recuperado de http://www.redalyc.org/pdf/1942/ 194222473010.pdf

16. Padilla, G., Leal, F., Hernández, M. y Cabero, J. (2012). Un reto para el profesor del futuro: la tutoría virtual. México: SINED. Recuperado de file://C:/Users/msotelo/Downloads/42-3-56-1-10-20140623.pdf 
17. Imbernón, F., Silva, P., y Guzmán, C. (2011). Competencias en los procesos de enseñanza-aprendizaje virtual y semipresencia. Revista Científica de Educomunicación. Comunicar , XVIII(36), 107-114. Recuperado de: http://www.revistacomunicar. com/index. php?contenido $=$ detalles\&numero $=36 \&$ articulo $=36-2011-13$

18. Moreno, F. (Octubre, 2011). ¿Qué es ser un buen profesor o una buena profesora del Instituto Cervantes? Análisis de las creencias del alumnado, profesorado y personal técnico y directivo de la institución. (Informe de Investigación). Recuperado de: http://cfp.cervantes.es/imagenes/File/recursos_proyectos/informe_bue n_profesor_ele/informe-buen-profesor-cervantes.pdf

19. López, A., González, I., y De León, C. (2014). Perfil de un buen docente. Aplicación de un protocolo de evaluación de las competencias del profesorado universitario. Revista Electrónica Interuniversitaria de Formación del Profesorado, 17 (1), 133-148. Recuperado

de: http://www.aufop.com/aufop/uploaded_files/articulos/ 1400802026.pdf

20. Organización de las Naciones Unidas para la Educación, la Ciencia y la Cultura [UNESCO]. (2008). Estándares de competencia en TIC para docentes. Londres: UNESCO. Recuperado de http://www.oei.es/tic/UNESCOEstandaresDocentes.pdf

21. Suárez, M., y Fernández, M. (2013). La enseñanza Virtual y el nuevo perfil de profesor: renovarse o no estar a la altura. Trabajo presentado en las IV Jornadas Internacionales de Campus Virtuales. España. Recuperado de: http://campusvirtuales2013.uib.es/ docs/157.pdf

22. Vera-Noriega, J.A., Pimentel, C. E.,y Batista, F.J. (2005). Redes semánticas: aspectos teóricos, técnicos, metodológicos y analíticos. $\mathrm{Ra}$ Ximhai, 1(3). Recuperado de http://www.redalyc .org/articulo.oa?id=46110301 